\title{
Razones a favor de una interpretación material del condicional*
}

\section{Reasons in favor of a material interpretation of the conditional}

\author{
Miguel López-Astorga ${ }^{* *}$ \\ ORCID: 0000-0002-6004-0587 \\ Universidad de Talca, Chile
}

Recibido: 23 de agosto de 2017

Revisado: 1 de octubre de 2017 Aceptado: 2 de diciembre de 2017

\section{Resumen}

Desde la antigüedad se ha discutido cuál es la forma más apropiada en la que debemos entender los enunciados condicionales. El debate, de hecho, llega hasta nuestros días y dos teorías cognitivas contemporáneas, la teoría de la lógica mental y la teoría de los modelos mentales, presentan visiones encontradas al respecto. Según la primera de ellas, el condicional, sin duda, no es material, pero, aunque no lo afirma explícitamente, la segunda parece atribuirle inicialmente características consistentes con tal interpretación. En este trabajo, se trata de profundizar en esta polémica y demostrar que los ejemplos de condicional ofrecidos por la teoría de la lógica mental con el propósito de evidenciar que una comprensión material de los mismos no es adecuada. No obstante, si son analizados detenidamente bajo el prisma de la teoría de los modelos mentales, se presenta una comprensión de las oraciones condicionales en tal dirección. 
Palabras clave: condicional; interpretación material; lógica clásica; lógica mental; modelos mentales

\begin{abstract}
The most suitable way to understand conditional sentences has been discussed since antiquity. Two contemporary cognitive theories, mental logic theory and mental models theory, have opposite views in that regard. According to the former, the conditional is undoubtedly not material. Although it does not explicitly state it, the latter seems, in principle, to attribute characteristics consistent with that interpretation to this operator. In this paper, this controversy is viewed in depth and an attempt is made to show that examples of conditionals given by the mental logic theory in order to demonstrate that a material understanding of them is not right can reveal, nevertheless, if analyzed in detail from the perspective of the mental models theory, that the average individual does tend to an understanding of conditional sentences in that direction.
\end{abstract}

Keywords: conditional; material interpretation; classical logic; mental logic; mental models

\section{Introducción}

Cuál es la manera más apropiada de entender los enunciados condicionales ha sido un asunto que ha generado importantes controversias desde la antigua Grecia. Ciertamente, fuentes como los textos Adversus Mathematicos y Pyrrhoneae Hypotyposes, ambos escritos por Sexto Empírico, nos revelan que en le siglo IV a. C. se pudo asistir a una intensa disputa al respecto (véase por ejemplo O'Toole \& Jennings, 2004). No obstante, el aspecto más interesante de tal debate para este trabajo es que una de las posiciones que se defendieron en él fue la de Filón de Megara, quien entendió el condicional de un modo material, esto es, como lo entiende la lógica estándar en nuestros días (véase por ejemplo al margen de las obras de Sexto mencionadas, Bocheński, 1963; O'Toole \& Jennings, 2004,).

Tal manera de entender el condicional consiste en considerar que tal conectiva solo puede ser falsa en un caso: cuando su primer término es verdadero y el segundo es falso. En todos los demás casos, a saber, cuando ambos términos son verdaderos, cuando el primero es falso y el segundo verdadero y cuando los dos son falsos, la sentencia condicional globalmente considerada es verdadera. Así, una manera más formal de expresar esta misma idea puede ser asumir que ' $v(X)$ ' hace referencia la valor de verdad de $X$, que ' 1 ' denota verdad, que ' 0 ' expresa falsedad y que ' $X \rightarrow Y$ ' representa una relación condicional del tipo 'si $X$, entonces $Y$ ', y afirmar que:

$v(X \rightarrow Y)=0$ si y solo si $v(X)=1$ y $v(Y)=0$. De lo contrario, $v(X \rightarrow Y)=1$.

0 , si se prefiere, que

$v(X \rightarrow Y)=1$ en tres casos:

$$
\begin{aligned}
& \text { - Cuando } v(\mathrm{X})=1 \text { y } v(\mathrm{Y})=1 . \\
& \text { - Cuando } v(\mathrm{X})=0 \text { y } v(\mathrm{Y})=1 . \\
& \text { - Cuando } v(\mathrm{X})=0 \text { y } v(\mathrm{Y})=0
\end{aligned}
$$

En el caso restante, $v(X \rightarrow Y)=0$ (análisis semejantes de los valores de verdad del condicional pueden encontrarse también en diferentes trabajos, por ejemplo, López-Astorga, 2016a).

Pero el caso es que, del mismo modo que lógicos griegos como Crisipo de Solos o Diodoro Cronos pusieron en cuestión esta propuesta de Filón ( 0 'Toole y Jennings, 2004,), una teoría contemporánea, la teoría de la lógica mental (Bompastor Borges Dias \& Roazzi, 2003; Braine \& O’Brien, 1998a; Gouveia, Roazzi, Moutinho, Bompastor Borges Dias \& O’Brien, 2002; Gouveia, Roazzi, O'Brien, Moutinho, \& Bompastor Borges Dias, 2003; O'Brien, 
2009, 2014; O’Brien \& Li, 2013; O’Brien \& Manfrinati, 2010), a pesar de que plantea que, tanto el pensamiento como el lenguaje, están vinculados a una lógica formal y a una 'sintaxis del pensamiento', también defiende que la interpretación material del condicional, es decir, como se ha dicho, la que atribuye a esta última conectiva la lógica clásica, no es la que corresponde ni a tal lógica ni a tal sintaxis, ni tampoco, por tanto, al modo habitual en que las personas suelen entender los enunciados en los que aparecen los términos 'si' y 'entonces'. Así, se puede decir que la polémica continúa, de alguna forma abierta en el presente, especialmente si se tiene en cuenta que la teoría de la lógica mental no es la única teoría cognitiva en la actualidad y que otra teoría rival, la de los modelos mentales semánticos, propone una visión alternativa del condicional que, en cierta medida y al menos inicialmente, recuerda la de Filón (Hinterecker, Knauff \& Johnson-Laird, 2016; Johnson-Laird, 2010, 2012, 2015; Johnson-Laird \& Byrne, 2002; Johnson-Laird, Girotto \& Legrenzi, 1999; Johnson-Laird, Khemlani \& Goodwin, 2015; Orenes \& Johnson-Laird, 2012; Ragni, Sonntag \& Johnson-Laird, 2016).

Efectivamente, la teoría de los modelos mentales, aunque no asume explícitamente la interpretación material del condicional y rechaza la existencia de relaciones claras entre la actividad intelectual humana y la lógica estándar, como se indica más adelante, parece atribuirles a las oraciones condicionales características que, aparentemente, coinciden con las que Filón de Megara asigna a tal tipo de enunciados. En este sentido, el objetivo fundamental de en este texto es profundizar en esta discusión contemporánea y mostrar que la concepción del condicional defendida por la teoría de los modelos mentales parece ajustarse más al modo real en que las personas tienden a entenderlo y que, por tanto, aún no podemos descartar la posibilidad de que la interpretación más adecuada para este operador sea una no muy distante de la material.

Para trabajar en esta dirección se van a analizar, en primer lugar, ejemplos de proposiciones condicionales presentados por la teoría de la lógica mental con el propósito de mostrar que no resisten, de ninguna manera, una interpretación como la que establece la lógica clásica. El fin de tal análisis es, evidentemente, argumentar que el motivo por el que tales ejemplos son problemáticos no es que la interpretación material sea ajena a la mente humana, sino, por el contrario, que los individuos habitualmente los entienden de la manera señalada por la teoría de los modelos mentales, esto es, como se ha reflejado, de una manera bastante próxima a tal interpretación. El propósito básico será, de esta forma, tratar de mostrar que las cláusulas de esos ejemplos incluyen contenidos semánticos para los que no es adecuada una relación condicional material, es decir, una relación que solo conduce a falsedad cuando la primera cláusula (o antecedente) es verdadera y la segunda (o consecuente) es falsa, y que las personas suelen rechazarlos precisamente porque, dado que suelen entender el condicional materialmente, notan este punto.

Así, este trabajo se articula en dos grandes apartados. En el primero de estos, se exponen las líneas generales de la posición de la teoría de la lógica mental sobre el condicional y, como se ha apuntado, algunos de los ejemplos emblemáticos que sus defensores suelen proponer para apoyar tal posición. En el segundo, por su parte, se revisarán esos mismos ejemplos desde el margo global de la teoría de los modelos mentales con la intención de evidenciar que los seres humanos suelen comprenderlos como esta última teoría predice, y que esta es exactamente la razón por la que son controvertidos. Las líneas siguientes versan sobre la teoría de la lógica mental y sus ejemplos.

\section{El condicional de la lógica mental}

Como se ha mencionado, la teoría de la lógica mental defiende la idea de que la mente humana opera de manera lógica y se atiene a una sintaxis universal. No obstante, como igualmente se ha reflejado, tal lógica no es, ni mucho menos, la lógica clásica tal y como está descrita en trabajos como el de Deaño (1999), ni, por consiguiente, es necesariamente consistente con cálculos como el de Gentzen (1934, 1935). La teoría de la lógica mental es una teoría que se forja, esencialmente, a partir de resultados empíricos, los cuales llevan 
a asumir como propias de la sintaxis del pensamiento exclusivamente aquellas reglas formales que, de acuerdo con tales resultados, las personas verdaderamente parecen utilizar. Es cierto que esta labor experimental ha conducido a la teoría a una serie de reglas que sonválidas en el cálculo proposicional estándar (Braine \& O’Brien, 1998b,), pero esto no implica la relación inversa. Esto es, que la teoría de la lógica mental acepte la totalidad de los esquemas formales que son válidos en dicho cálculo. Por lo demás, hay otros puntos importantes en los que la teoría de la lógica mental se distancia de la lógica clásica y uno de estos es, claramente, la manera en la que considera los enunciados condicionales.

Dado que este último aspecto de la teoría es el que es relevante para este trabajo, se van a obviar aquí todas las demás tesis a las que se podría hacer referencia y nos vamos a centrar en él. Para sus partidarios, la interpretación de Filón de Megara del condicional no es aceptable por los mismos motivos que expusieron ya sus detractores en la Grecia clásica, a saber, porque lleva a tener que admitir oraciones absurdas (Bompastor Borges Dias \& Roazzi, 2003). Tales oraciones absurdas son posibles bajo la interpretación material, debido a que esta última, permite vincular formalmente contenidos que el conocimiento general puede considerar absolutamente dispares y sin conexión por medio de una relación condicional. No obstante, en realidad,

“... se indica suposição, e quando a pessoa está raciocinando, geralmente supõe algo para descobrir como seria se esta suposição fosse verdadeira. 0 raciocínio condicional envolve situações hipotéticas, de faz-de-conta, e o que se segue a partir dessas situações. Não é racionar apenas em termo de relação formal entre as premissas, sem levar em consideração o valor-verdade empírico"1 (Bompastor Borges Dias y Roazzi, 2003, pp. 47-48, cursivas en el texto original).

1 '...si indica suposición y, cuando la persona está razonando, generalmente supone algo para descubrir cómo sería si esa suposición fuese verdadera. El razonamiento condicional implica situaciones hipotéticas, fingidas, y lo que se sigue a partir de tales situaciones. No es razonar simplemente en términos de una relación formal entre las premisas, sin tomar en consideración el valor de verdad empírico'.
Por tanto, la objeción de la teoría de la lógica mental contra la propuesta de Filón de Megara, sin ser necesariamente la misma, recuerda la atribuida a Crisipo de Solos contra esa misma concepción del condicional (Gould, 1970; O’Toole \& Jennings, 2004). Y esto es así porque lo que se está manifestando es que debe existir una relación conceptual entre el antecedente y el consecuente, 0 , si se prefiere, una conexión semántica entre ambos. De lo contrario, pueden darse paradojas bastante llamativas (Bompastor Borges Dias \& Roazzi, 2003, O’Brien, 2014).

Así, algunos ejemplos de enunciados absurdos que son válidos de acuerdo con la interpretación material y que, sin embargo, el individuo promedio difícilmente puede aceptar pueden ser los siguientes:

[1]: "Se dois é um número par, então Recife é a capital de Pernambuco"2 (Gouveia et al., 2002, p. 218).

[2]: "Se dois não é um número par, então Recife é a capital de Pernambuco"3 (Gouveia et al., 2002, p. 218).

[3]: "Se dois não é um número par, então Recife não é a capital de Pernambuco"4 (Gouveia et al., 2002, p. 218).

[4]: "If 4 is a prime number then the Queen of Scotland authored this article"5 (O'Brien, 2014, p. 223).

Sin duda, como propugnan los defensores de la teoría de la lógica mental, la mayor parte de las personas tenderá a rechazar enunciados como estos. No obstante, si el criterio para evaluarlos es el criterio material de la lógica estándar, los cuatro son claramente verdaderos, y esto es muy fácil de comprobar. Evidentemente, si $\mathrm{X}$ representa el hecho de que dos es un número par e $Y$ el hecho de que Recife es la capital de Pernambuco, [1]

2 'Si dos es un número par, entonces Recife es la capital de Pernambuco'.

3 'Si dos no es un número par, entonces Recife es la capital de Pernambuco'.

4 'Si dos no es un número par, entonces Recife no es la capital de Pernambuco'.

5 'Si 4 es un número primo, entonces la reina de Escocia es la autora de este artículo'. 
es verdadero porque, como sabemos, $v(X)=1 \mathrm{y}$ $v(Y)=1$. Por su parte, [2] también es correcto porque, en este caso, $v(\neg X)=0$ y $v(Y)=1$ (donde ' $\neg$ ' hace referencia a la negación lógica). De igual manera, [3] es válido porque los valores son ahora $v(\neg X)=0$ y $v(\neg Y)=0$.

Por último, con respecto a [4], se puede decir que, si en este ejemplo $X$ denota la situación en que cuatro es un número primo e $Y$ la situación en que la reina de Escocia es la autora de este artículo (obviamente, O'Brien se refiere al artículo escrito por él en 2014 y en el que se incluyó [4]), la oración también es verdadera, ya que se trata de un caso en el que $v(\mathrm{X})=0$ y $v(\mathrm{Y})=0$.

Tenemos, por tanto, que [1] es verdadero porque tanto su antecedente como su consecuente lo son, que [2] también lo es porque su antecedente es falso y su consecuente es verdadero y que [3] y [4] lo son igualmente porque en estos ambas cláusulas son falsas. De esta manera, ninguno de los ejemplos mencionados representaría un caso de antecedente verdadero y consecuente falso, es decir, un caso en el que el condicional completo tendría que ser considerado falso, y, por esta razón, si la interpretación natural de este operador fuera la material, como se ha dicho, las personas tendrían que admitirlos como correctos.

Empero, los partidarios de la teoría de la lógica mental sostienen que la única manera en que proposiciones como las indicadas pueden ser aceptadas por un individuo es que se hallen inmersas en un contexto pragmático que relativice su carácter condicional. Tal idea se basa en que, para ellos, la presencia de los términos 'si' y 'entonces' no garantiza que una oración sea interpretada como un condicional y un enunciado puede ser entendido como un condicional aunque no incluya tales términos (Gouveia et al., 2002). En este sentido, [3], por ejemplo, podría ser admitido solo si formara parte de un diálogo como este:

[5]: “A: Dois não é um número par!

B: Claro que dois é um número par.

A: Não é.
B: Se dois não é um número par, então Recife não é a capital de Pernambuco"6 (Gouveia et al., 2002, p. 218).

De igual forma, no es difícil pensar en un contexto pragmático que haga también admisible, por ejemplo, a [4]:

[6]: A: ¡Cuatro es un número primo!

B: En absoluto. No lo es.

A: Por supuesto que sí.

B: Si cuatro es un número primo, entonces la reina de Escocia es la autora de este artículo.

Sin embargo, por sí solos, sin mayor información contextual acompañándolos, [1], [2], [3] y [4] serían como mínimo, considerados absurdos por los individuos. Este es, como se ha señalado, el principal argumento de la teoría de la lógica mental contra la interpretación material, una interpretación que, según este enfoque, si fuera realmente la natural en la mente humana, llevaría a la aceptación sin reservas y absoluta de esos mismos ejemplos.

Existe, no obstante, otra posibilidad teórica. La propuesta de la lógica mental puede ser ignorada y se puede asumir otro enfoque contemporáneo mucho más semántico, el de la teoría de los modelos mentales. Si se procede de esta forma, se puede comprobar que el motivo por el que enunciados como [1], [2], [3] y [4] son habitualmente rechazados es, precisamente, que las personas presentan la tendencia a interpretar los condicionales como indicó Filón. De hecho, esta última teoría permite también entender qué es lo que sucede en la mente en contextos pragmáticos como los de [5] y [6] y, por tanto, conocer las razones exactas por las que, en casos como esos, las sentencias sí son aceptadas. Todo esto se trata de explicar en el siguiente apartado.

6 'A: ¡Dos no es un número par!

B: Claro que dos es un número par. A: No es.

B: Si dos es un número par, entonces Recife no es la capital de Pernambuco'. 


\section{La teoría de los modelos mentales y las posibilidades semánticas del condicional}

El planteamiento básico de la teoría de los modelos mentales es radicalmente opuesto al de la teoría de la lógica mental. De acuerdo con la primera, las oraciones en lenguaje natural no remiten a formas lógicas sintácticas, sino a modelos semánticos que consisten en representaciones icónicas de la realidad. Esto significa que tales modelos

... hanno una struttura che corrisponde alla struttura di ciò che essi rappresentano. Di conseguenza, i modelli mentali sono affini ai modelli delle opere architettoniche, ai modelli di molecole complesse della biologia molecolare, ai diagrammi con cui in fisica si rappresentano le interazioni tra particelle ${ }^{7}$ (Johnson-Laird et al., 1999, p. 63).

Del mismo modo, en un texto más reciente, Johnson-Laird afirma que "... A mental model has a structure that corresponds to the known structure of what it represents"8 (Johnson-Laird, 2012, p. 136). Por tanto, queda claro que los modelos son estructuras simbólicas que describen la realidad de un modo bastante fiel y que, por esto, difícilmente pueden ser comparados con simples casos en una tabla de verdad de la lógica estándar. Sin embargo, a pesar de que esto es indiscutiblemente así, los modelos que inicialmente la teoría asigna a los distintos conectores tradicionales en lógica coinciden realmente con las combinaciones en las que tales conectores son verdaderos en sus tablas correspondientes. Esto es de este modo en todos los casos (véase, p.ej., Johnson-Laird, 2012, p. 138, Tabla 9.2), pero, puesto que este trabajo versa principalmente sobre el condicional, únicamente se exponen aquí los modelos que la teoría atribuye a este último operador. De esta manera, tales modelos, en el caso de una oración como 'si

7 '... tienen una estructura que corresponde a la estructura de lo que se representa. Por consiguiente, los modelos mentales son afines a los modelos de las obras arquitectónicas, a los modelos de las moléculas complejas de la biología molecular, a los diagramas con los que en física se representan las interacciones entre partículas'.

8 '... Un modelo mental tiene una estructura que se corresponde con la estructura conocida de lo que representa'.
$X$, entonces $Y$ ', son los que se indican a continuación:

[7]: X, Y

[8]: $\neg X, Y$

[9]: $\neg X, \neg Y$

Evidentemente, [7] representa una situación en la que las dos cláusulas suceden, [8] hace referencia a otra en la que solo tiene lugar el consecuente y [9] muestra un escenario en el que ninguna de las dos cláusulas es verdad. Y esto significa que la única situación que no está permitida es aquella en la que el antecedente acontece y el consecuente no, lo que revela, a su vez, un claro paralelismo con la tabla de verdad del condicional en la lógica clásica. Efectivamente, en [7], $v(X)=1 \mathrm{y}$ $v(\mathrm{Y})=1$, en [8], $v(\mathrm{X})=0 \mathrm{y} v(\mathrm{Y})=1 \mathrm{y}$, en [9], $v(\mathrm{X})=0 \mathrm{y}$ $v(Y)=0$. Por su parte, la única situación no permitida es, obviamente, una situación en la que $v(\mathrm{X})=1$ y $v(\mathrm{Y})=0$ (correspondencias entre los valores de la tabla de verdad del condicional y los modelos que la teoría de los modelos mentales asigna a esta conectiva pueden hallarse también, p. ej., en López-Astorga, 2014).

Sin embargo, aunque esto es así, los defensores de la teoría de los modelos mentales no suelen aceptarlo explícitamente. De hecho, a menudo tratan de hacer todo lo contrario, señalando características de los modelos que los distancian indiscutiblemente de los casos de las tablas de verdad. Tales características son varias, pero, quizás, la más relevante para los propósitos de este texto es que los modelos se ven sujetos a veces a procesos de modulación. La modulación es "... the process in the construction of models in which content, context, or knowledge can prevent the construction of a model and can add information to a model" 9 (Johnson-Laird et al., 2015, p. 202). No obstante, cómo funciona verdaderamente la modulación será explicado más adelante. De momento, lo que nos interesa es que no cabe duda de que, aunque como se ha dicho, los partidarios de la teoría de los modelos mentales tratan habitualmente de

9 '... el proceso en la construcción de modelos en el que el contenido, el contexto o el conocimiento pueden evitar la construcción de un modelo y pueden añadir información a un modelo'. 
alejarse todo lo posible de los principios y exigencias de la lógica estándar, no lo logran del todo en todos los aspectos, siendo una prueba de esto que los modelos que en ocasiones se asignan a las oraciones, coinciden, como se ha mostrado con el ejemplo del condicional, con las combinaciones en las que, en una tabla de verdad, tales oraciones serían verdaderas (este punto se desarrolla también en otros trabajos, p. ej., véase, con respecto a la disyunción, López-Astorga, 2015).

Sin embargo, en el caso concreto del condicional, esto, como también se ha reflejado, se puede traducir en que la teoría de los modelos mentales parece partir inicialmente de la interpretación material. Esta idea es fundamental, ya que si asumimos dicha teoría, puede revelarnos que los motivos por los que generalmente no se aceptan enunciados como [1], [2], [3] y [4] están vinculados, precisamente, con el hecho de que el ser humano manifiesta una tendencia a interpretar materialmente el condicional. La clave en este sentido reside en los experimentos que, con base en el marco general de los modelos mentales, realizaron Orenes y Johnson-Laird (2012). Algunos de tales experimentos apuntaban a demostrar que, efectivamente, una oración como 'si $X$, entonces $Y$ ' hace referencia, en principio, a los modelos [7], [8] y [9], y su manera de proceder fue sencilla.

En lógica estándar, de una fórmula tal como por ejemplo $Y$, se puede derivar $X \rightarrow Y$, y el motivo de esto es evidente: $X \rightarrow Y$ es siempre verdad, como se ha indicado más arriba, cuando $Y$ también lo es. No obstante, según Orenes y Johnson-Laird (2012), las personas no deberían aceptar tal inferencia, ya que el conjunto de posibilidades semánticas a las que hace referencia $X \rightarrow Y$ incluye [9], esto es, un escenario en el que la premisa $Y$ es falsa. De este modo, diseñaron para una de sus condiciones experimentales una serie de ejercicios con contenido temático cuya estructura formal era exactamente: 'Dado $\neg Y$, ¿se sigue que, si $X$, entonces $\neg Y$ ?', y los resultados que obtuvieron confirmaron su predicción, ya que la mayor parte de sus participantes consideró ese tipo de inferencia incorrecto.
No obstante, para confirmar aún más sus supuestos, Orenes y Johnson-Laird (2012) pensaron también en otra condición experimental. En su opinión, si construían tareas en las que la conclusión con la oración condicional no pudiera, en virtud de un proceso de modulación, remitir al modelo problemático, la inferencia tendría que ser aceptada. De esta manera, pensaron igualmente en inferencias de tal tipo y se las propusierona sus participantes. Una de estas fue:

[10]: "Maria didn't listen to pop music. Does it follow that if Maria listened to music then she didn't listen to pop music?"10 (Orenes y Johnson-Laird, 2012, p. 375).

La particularidad de esta tarea es que su condicional no se puede vincular con tres posibilidades, sino solo con dos, las cuales son, si tenemos en cuenta la negación que aparece en el consecuente, estas:

[11]: María escuchó música, María no escuchó música pop

[12]: María no escuchó música, María no escuchó música pop

Claramente, falta el modelo en el que María no escucha música y escucha música pop, pero, dado que tal escenario es imposible (si se escucha música pop, necesariamente se escucha música), la modulación lo elimina. Sin embargo, lo que es relevante aquí es que, puesto que ahora no hay ningún modelo contradictorio con la premisa, esto es, ningún modelo en que María escuche música pop, una inferencia de este tipo tendría que ser admitida como válida por el individuo promedio. $\mathrm{Y}$, ciertamente, los resultados logrados por Orenes y Johnson-Laird (2012) confirmaron este punto, ya que sus participantes sí tendieron a aceptar este segundo tipo de inferencia, hecho que, sin duda, apoyan también los postulados esenciales de la teoría.

Empero, desde esta óptica, está claro que los ejemplos tomados de la literatura sobre la teoría de la lógica mental, esto es, [1], [2], [3] y [4],

$\overline{10}$ 'María no escuchó música pop. ¿Se sigue que, si María escuchó música, entonces no escuchó música pop?' 
son, oraciones con modelos contradictorios con una premisa, aunque esta sea implícita y proceda del conocimiento general del individuo. Así, en el caso de [1], se puede decir que sus modelos son:

[13]: Dos es un número par, Recife es la capital de Pernambuco

[14]: Dos no es un número par, Recife es la capital de Pernambuco

[15]: Dos no es un número par, Recife no es la capital de Pernambuco

[13] no presenta ningún problema, pero [14] es inconsistente con el hecho conocido de que dos es realmente un número par y [15] con ese mismo hecho y con el dato de que Recife sí es la capital de Pernambuco.

Del mismo modo, en [2], por su parte, estaríamos hablando de estas combinaciones de posibilidades:

[14]: Dos no es un número par, Recife es la capital de Pernambuco

[13]: Dos es un número par, Recife es la capital de Pernambuco

[16]: Dos es un número par, Recife no es la capital de Pernambuco

Como se ha explicado en el caso anterior, si bien [13] no crea dificultades, [14] es incompatible con una información sabida. Además, ahora hay que añadir [16], que es incoherente con el hecho real de que Recife es la capital de Pernambuco.

Tras estos argumentos, el caso de [3] parece bastante simple de entender. Sus escenarios posibles son:

[15]: Dos no es un número par, Recife no es la capital de Pernambuco

[16]: Dos es un número par, Recife no es la capital de Pernambuco

[13]: Dos es un número par, Recife es la capital de Pernambuco
Como ya se ha indicado, [15] y [16] serían casos problemáticos. Por último, los modelos de [4] corresponden a este conjunto:

[17]: Cuatro es un número primo, La reina de Escocia es la autora de este artículo

[18]: Cuatro no es un número primo, La reina de Escocia es la autora de este artículo

[19]: Cuatro no es un número primo, La reina de Escocia no es la autora de este artículo

Aquí, [19] es la única posibilidad aceptable, ya que presenta dos informaciones evidentes. Sin embargo, [17] es inconsistente con el dato transmitido por el conocimiento general de que cuatro no es un número primo y con el hecho indiscutible de que la autora del artículo no es la reina de Escocia. Del mismo modo, [18] es también inconsistente con este último hecho.

Vemos por tanto, que los cuatro ejemplos cuentan con modelos inaceptables por su incompatibilidad con el conocimiento general o con lo que es sabido, lo que, por supuesto, puede ser una clara explicación de por qué oraciones como estas no suelen ser admitidas. Pero, como se indicó antes, si asumimos esta explicación, estamos asumiendo también que la mente humana tiende a interpretar los condicionales, al menos al principio y cuando no intervienen procesos de modulación, materialmente. Esto es así porque, como también se ha señalado, aunque sean representaciones icónicas con características que los distinguen de las variables proposicionales, las posibilidades semánticas que corresponden en un primer momento al condicional coinciden con las situaciones en las que, según Filón de Megara, tal operador es verdadero.

Por lo demás, al margen de los hallazgos de Orenes y Johnson-Laird (2012) comentados antes, otros argumentos también apoyan la propuesta de la teoría de los modelos mentales. Tales argumentos apuntan a que esta teoría cuenta igualmente con la maquinaria necesaria para explicar de modo preciso lo que sucede en contextos pragmáticos como los de [5] y [6]. Como se muestra en Johnson-Laird y Byrne (2002), aunque los modelos 
que de entrada pueden ser asignados a un condicional son, efectivamente, [7], [8] y [9], hasta nueve conjuntos de combinaciones más son posibles para esta conectiva (véase Johnson-Laird \& Byrne, 2002, p. 663, Tabla 4). Son, por consiguiente, diez las combinaciones de posibilidades que pueden corresponder a un condicional y cuál de ellas se vincula a una expresión concreta con la estructura 'si... entonces...' depende, obviamente, de procesos de modulación. Esto sin duda tiene diversas implicaciones, pero la más importante es que se pueden describir los procesos mentales concretos por los que el sentido de un condicional puede verse modificado en unas circunstancias determinadas.

Efectivamente, de lo expuesto previamente, puede deducirse que, en virtud de la modulación, elementos como el significado concreto de los términos empleados en las proposiciones y el contexto en el que las oraciones son utilizadas pueden conducir a la modificación o la eliminación de algunos de los modelos iniciales de un enunciado. Así, por lo que respecta a [5] y [6], lo que nos interesa es que algunas de las diez interpretaciones finales indicadas por Johnson-Laird y Byrne (2002) tienen solo un modelo. Tal es el caso de interpretaciones como las denominadas por estos 'Ponens' y 'Tollens'. Uno de sus ejemplos de Ponens es el siguiente:

[20]: "If my name is Alex then Viv is engaged" 11 (Johnson-Laird \& Byrne, 2002, p. 663, Tabla 4).

Evidentemente, lo que [20] quiere decir es que el hecho de que Viv está comprometida es tan cierto como el hecho de que el nombre de la persona que habla es Alex y, de esta manera, la frase solo parece hacer referencia a este modelo:

[21]: Mi nombre es Alex, Viv está comprometida

Todas las demás opciones quedan eliminadas por factores semánticos, contextuales o pragmáticos. $Y$ es que lo que sucede es que se supone que el nombre de la persona que afirma la oración es

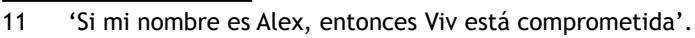

realmente Alex y que lo que quiere transmitir es que la única posibilidad en el universo es que, además de que su nombre sea el indicado, Viv esté comprometida.

Por su parte, uno de los ejemplos que ofrecen de Tollens es este:

[22] "If it works then I'll be obligated to jump in the lake"12 (Johnson-Laird \& Byrne, 2002, p. 663, Tabla 4).

También es evidente lo que esta oración pretende transmitir: algo (aquello a lo que se refiere el antecedente) no va a funcionar y yo no voy a saltar en el lago, y tan seguro es un hecho como el otro. Por tanto, el único modelo aquí es:

[23]: No funciona, Yo no salto en el lago

La pragmática, la semántica y el contexto también eliminan aquí otras posibles alternativas. $Y$ esto sucede porque no existe la más mínima duda de que lo que el hablante quiere decir es que ni aquello a lo que se refiere va a funcionar ni él va a saltar en el lago.

No obstante, si esto es de este modo, verdaderamente queda claro lo que acontece tanto en [5] como en [6]. En [5], el contexto de la discusión muestra que lo que en realidad quiere decir al final $B$ con su última afirmación es que dos tiene que ser necesariamente un número par, y que ello es tan evidente que, si se niega, hay que negar también que Recife sea la capital de Pernambuco. Es decir, que el único modelo que se puede atribuir al condicional final es [13], el cual es un caso de Ponens y describe una situación en la que solo es posible la paridad de dos y un estado de Pernambuco con su capital en Recife.

Y algo similar sucede con [6]. En este caso, lo que se pretende afirmar es que, si aceptamos que cuatro es un número primo, tenemos que aceptar también que la autora del artículo es la reina de Escocia, esto es, que ni cuatro es un número primo ni la reina de Escocia es la autora del artículo, lo cual queda reflejado en un modelo como [19],

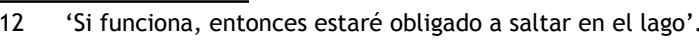


el único que se puede vincular al condicional con el que finaliza el diálogo de [6], el cual, como se puede apreciar, no es más que un caso de Tollens.

Por tanto, parece cierto lo que se ha señalado antes. El aparato conceptual de la teoría de los modelos mentales permite ir más allá que la teoría de la lógica mental y mostrar de un modo más nítido cómo son los procesos intelectuales específicos que pueden llevar a diferentes interpretaciones de los condicionales. La teoría de la lógica mental parece reconocer, como se ha indicado, que diversas interpretaciones son posibles pero no cabe duda de que la teoría de los modelos mentales profundiza más en esta dirección.

Contamos de esta manera, con diversas razones para decantarnos por la teoría de los modelos mentales. No obstante, si lo hacemos, y esto es precisamente lo que se ha intentado mostrar a lo largo del texto, tenemos que reconocer también que la interpretación material es la que, en un primer momento, o, si se prefiere por defecto, las personas suelen atribuir al condicional.

\section{Conclusiones}

Ya se mencionó anteriormente que esta no es precisamente la intención de los partidarios de la teoría de los modelos mentales. En general, ellos tratan de alejarse de la lógica clásica y un elemento característico por antonomasia de tal lógica, como lo demuestran sus tablas de verdad, es la interpretación material del condicional. Sin embargo, la coincidencia entre los modelos básicos de este operador lógico y sus valores de verdad en la lógica estándar nos ha permitido, al menos, observar una tendencia inicial a la interpretación material en la mente humana.

Discusiones en esta línea pueden ser encontradas también en diversos trabajos (por ejemplo, LópezAstorga, 2016b). Sin embargo, lo que aquí merece la pena destacar es que, como también lo hacen en algunas ocasiones tales trabajos, parece que lo deseen o no sus defensores, la teoría de los modelos mentales se solapa en ciertas ocasiones con la lógica clásica y, de alguna manera, no logra escapar de esta última por completo. Es evidente que este hecho nos abre un camino que, aunque no sea del tipo que los autores que apoyan la teoría de los modelos mentales desean habitualmente transitar, puede arrojarnos importantes luces sobre las verdaderas relaciones existentes entre la lógica, nuestro lenguaje y nuestro entendimiento.

Sobre este último punto, podemos decir que, si bien la literatura de la ciencia cognitiva viene demostrando con claridad, que la actividad mental humana no es una actividad puramente lógica y que hay elementos semánticos y pragmáticos que son determinantes en algunos procesos intelectuales, también se pueden apreciar en dicha literatura, al mismo tiempo, ciertas conexiones entre nuestra actividad inferencial y aspectos básicos de la lógica. Una de tales conexiones se ha evidenciado a lo largo de estas páginas, las cuales nos han revelado, como se ha expuesto, que, incluso asumiendo una perspectiva, como la de la teoría de los modelos mentales, que defiende la idea de que nuestro razonamiento es ajeno a la lógica, parece inevitable continuar considerando tendencias o inclinaciones compatibles con esta última.

Y esto, de la misma forma, no deja de ser curioso en otro sentido. A priori, podría pensarse acertadamente, que la teoría de la lógica mental, tiene muchos más puntos en común con la lógica clásica que la teoría de los modelos mentales aunque también se distancia de ella. Empero, precisamente en el caso de uno de los aspectos esenciales de la lógica estándar que la teoría de la lógica mental rechaza, el de la interpretación propuesta por Filón, su teoría rival, la de los modelos mentales, parece mostrar que está errada. Por tanto, aunque es indudable que en el presente, cálculos como el de Gentzen $(1934,1935)$ no pueden ser aceptados como modelos descriptores del operar de la mente humana ${ }^{13}$ también lo es que no podemos tampoco rechazar de una manera radical todos los principios y reglas de la lógica clásica en cuanto elementos, al menos, condicionantes del pensamiento.

13 Esto lo demuestran, entre otros hechos, resultados como los obtenidos por Orenes y Johnson-Laird, 2012, comentados antes, ya que, en definitiva, como se ha señalado, de acuerdo con dicho cálculo, debería ser posible derivar una fórmula como $X \rightarrow Y$ a partir de una fórmula como $Y$, independientemente del contenido semántico de tanto $\mathrm{X}$ como $\mathrm{Y}$. 
Así, continuar con estudios como el presentado en este texto pueden ayudar, poniendo en tensión teorías cognitivas actuales, a descubrir en qué medida la lógica, si es que verdaderamente lo hace de manera significativa, incide en nuestra mente, hasta qué punto esto es así y en qué momentos se detiene para dar paso a otros recursos externos a ella que pueden estar también disponibles en nuestra maquinaria cognitiva. Las opciones, sin duda, son múltiples aún y la problemática no se encuentra cerrada todavía en absoluto. Es posible que lo que rige nuestro intelecto sea una lógica similar a la clásica, aunque con nítidos elementos diferenciadores con respecto a esta última (independientemente de que tal lógica comparta o no elementos más o menos comunes con el sistema propuesto por la teoría de la lógica mental).

Empero, otra posibilidad es que nuestra arquitectura cognitiva contenga recursos que, por su semejanza, recuerden a la lógica estándar a pesar de ser totalmente diferentes de esta (quizás, esta sería la posición de la teoría de los modelos mentales). Incluso, una tercera opción podría ser que las dos posibilidades anteriores fueran correctas al unísono (de hecho, trabajos como, por ejemplo, el de López-Astorga, 2015, tratan de establecer vínculos entre, precisamente, la teoría de la lógica mental y la de los modelos mentales). Quedan, en cualquier caso muchas incógnitas y, como se ha propuesto, un intento de empezar a superarlas puede ser trabajar en temáticas como la abordada en este trabajo.

\section{Referencias}

Bocheński, I. M. (1963). Ancient Formal Logic. Amsterdam, Holanda: North-Holland.

Bompastor Borges Dias, M. G., \& Roazzi, A. (2003). A teoria da lógica mental: $E$ os estudos empíricos em crianças e adultos. Psicologia em Estudo, 8(1), 45-55.

Braine, M. D. S., \& O’Brien, D. P. (Eds.) (1998a). Mental Logic. Mahwah, NJ: Lawrence Erlbaum Associates, Inc., Publishers.
Braine, M. D. S., \& O’Brien, D. P. (1998b). The theory of mental-propositional logic: Description and illustration. En M. D. S. Braine y D. P. O'Brien (Eds.), Mental Logic (pp. 79-89). Mahwah, NJ: Lawrence Erlbaum Associates, Inc., Publishers.

Deaño, A. (1999). Introducción a la lógica formal. Madrid, España: Alianza Editorial.

Gentzen, G. (1934). Untersuchungen über das logische Schließen I. Mathematische Zeitschrift, 39(2), 176-210.

Gentzen, G. (1935). Untersuchungen über das logische Schließen II. Mathematische Zeitschrift, 39(3), 405-431.

Gould, J. B. (1970). The Philosophy of Chrysippus. Albany, NY: State University of New York Press.

Gouveia, E. L., Roazzi, A., Moutinho, K., Bompastor Borges Dias, M. G., \& O'Brien, D. P. (2002). Raciocínio condicional: influências pragmáticas. Estudos de Psicologia, 7(2), 217-225.

Gouveia, E. L., Roazzi, A., O’Brien, D. P., Moutinho, K., \& Bompastor Borges Dias, M. G. (2003). Raciocínio dedutivo e lógica mental. Estudos de Psicologia, 20(3), 135-145.

Hinterecker, T., Knauff, M., \& Johnson-Laird, P. N. (2016). Modality, probability, and mental models. Journal of Experimental Psychology: Learning, Memory, and Cognition, 42(10), 1606-1620.

Johnson-Laird, P. N. (2010). Against logical form. Psychologica Belgica, 5(3/4), 193-221.

Johnson-Laird, P. N. (2012). Inference with mental models. En K. J. Holyoak y R. G. Morrison (Eds.), The Oxford Handbook of Thinking and Reasoning (pp. 134-145). Nueva York, NY: Oxford University Press.

Johnson-Laird, P. N. (2015). How to improve thinking. En R. Wegerif, L. Li y J. C. Kaufman (Eds.), The Routledge International Handbook of Research on Teaching Thinking (pp. 80-91). Abingdon, UK, y Nueva York, NY: Routledge. 
Johnson-Laird, P. N., \& Byrne, R. M. J. (2002). Conditionals: A theory of meaning, pragmatics, and inference. Psychological Review, 109(4), 646-678.

Johnson-Laird, P. N., Girotto, V., \& Legrenzi, P. (1999). Modelli mentali: Una guida facile per il profano. Sistemi Intelligenti, XI(1), 63-84.

Johnson-Laird, P. N., Khemlani, S., \& Goodwin, G. P. (2015). Logic, probability, and human reasoning. Trends in Cognitive Sciences, 19(4), 201-214.

López-Astorga, M. (2014). Mental models and syntactic rules: A study of the relations between semantics and syntax in inferential processes. Analele Universitatii din Craiova, Seria Filosofie, 33(1), 107-117.

López-Astorga, M. (2015). The disjunction introduction rule: Syntactic and semantic considerations. Pragmalingüística, 23, 141-149.

López-Astorga, M. (2016a). The problem of the negation of the conditional. Rupkatha, 8(4), 21-29.

López-Astorga, M. (2016b). Logic, pragmatics, and types of conditionals. Frontiers of Philosophy in China, 11(2), 279-297.

O’Brien, D. P. (2009). Human reasoning includes a mental logic. Behavioral and Brain Sciences, 32, 96-97.
O'Brien, D. P. (2014). Conditionals and disjunctions in mental-logic theory: A response to Liu and Chou (2012) and to López-Astorga (2013). Universum, 29(2), 221-235.

O’Brien, D. P., \& Li, S. (2013). Mental logic theory: A paradigmatic case of empirical research on the language of thought and inferential role semantics. Journal of Foreign Languages, 36(6), 27-41.

O’Brien, D. P., \& Manfrinati, A. (2010). The mental logic theory of conditional proposition. En M. Oaksford y N. Chater (Eds.), Cognition and conditionals: Probability and Logic in Human Thinking (pp. 39-54). Oxford, UK: Oxford University Press.

Orenes, I., \& Johnson-Laird, P. N. (2012). Logic, models, and paradoxical inferences. Mind \& Language, 27(4), 357-377.

O'Toole, R. R. y Jennings, R. E. (2004). The Megarians and the Stoics. En D. M. Gabbay y J. Woods (Eds.), Handbook of the History of Logic, Volume 1. Greek, Indian and Arabic Logic (pp. 397-522). Amsterdam, Holanda: Elsevier.

Ragni, M., Sonntag, T. y Johnson-Laird, P. N. (2016). Spatial conditionals and illusory inferences. Journal of Cognitive Psychology, 28(3), 348-365. 